\title{
Improved Performance of GaAsSb/GaAs SQW Lasers
}

\author{
N. Hossain, S. R. Jin and S. J. Sweeney* \\ Advanced Technology Institute, University of Surrey, Guildford, Surrey GU2 7XH, UK. \\ S. -Q. Yu ${ }^{1}$, S. R. Johnson, D. Ding and Y. -H. Zhang \\ MBE Group, Arizona State University, Tempe, Arizona 85287, USA \\ ${ }^{1}$ now at Department of Electrical Engineering, University of Arkansas, 3217 Bell Engineering, \\ Fayetteville, AR, 72701, USA
}

\begin{abstract}

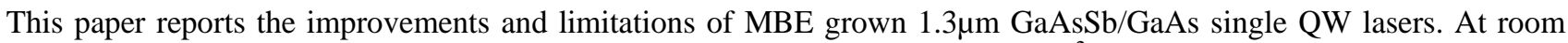
temperature, the devices show a low threshold current density $\left(J_{t h}\right)$ of $253 \mathrm{Acm}^{-2}$, a transparent current density of 98 $\mathrm{Acm}^{-2}$, an internal quantum efficiency of $71 \%$, an optical loss of $18 \mathrm{~cm}^{-1}$ and a characteristic temperature $\left(T_{0}\right)=51 \mathrm{~K}$. The defect related recombination in these devices is negligible and the primary non-radiative current path has a stronger dependence on the carrier density than the radiative current contributing to $\sim 84 \%$ of the threshold current at RT. From high hydrostatic pressure dependent measurements, a slight decrease followed by the strong increase in threshold current with pressure is observed, suggesting that the device performance is limited to both Auger recombination and carrier leakage.
\end{abstract}

Keywords: GaAsSb/GaAs, carrier recombination, quantum well laser.

\section{INTRODUCTION}

Semiconductor laser emitting $\sim 1.3 \mu \mathrm{m}$ are key for the rapid expansion of next generation metro-area networks. Vertical cavity surface-emitting lasers (VCSELs) operating at this wavelength have been of great interest due to their high performance and low cost manufacturing process. The conventional InGaAsP/InP QW material system used to make such lasers suffers from two problems: Firstly the poor thermal properties of InP-based lasers result in the need to incorporate sophisticated temperature control electronics into the package, leading to an order of magnitude increase in cost [1]. Secondly, due to the lack of lattice matching and high refractive index contrast materials to form all-epitaxial distributed Bragg reflectors (DBRs), it is also very difficult to fabricate monolithic VCSELs in the InP material system [2]. On the other hand, GaAs permits the growth of near lattice-matched GaAs/AlGaAs DBRs, which have superior optical and thermal properties when compared to other III-V DBRs [3]. Furthermore, the fabrication of GaAs based 1.3 $\mu \mathrm{m}$ VCSELs can take full advantage of the industrial standard $850 \mathrm{~nm}$ VCSEL fabrication technology, which is attractive from a manufacturing point of view. Lasers (edge emitting [4] and VCSELs [5]) based upon this material have been an active research area due to high potential for $1.3 \mu \mathrm{m}$ active regions on a GaAs substrate. The aim of this paper is to investigate the important recombination processes using temperature and pressure dependent measurements on edgeemitting lasers to aid in the design and optimization of GaAsSb/GaAs-based edge-emitting lasers and VCSELs.

\section{LASER STRUCTURE AND EXPERIMENTS}

In this study we investigated devices processed from wafers grown by Solid Source Molecular Beam Epitaxy. The active region consists of a $7 \mathrm{~nm} \mathrm{GaAs} \mathrm{nab}_{0.7} \mathrm{Sb}_{0.3} \mathrm{QW}$ within $15 \mathrm{~nm}$ GaAs spacers. The devices were measured as-cleaved with different cavity lengths. Further details on the growth and fabrication can be found in [6, 7]. Temperature dependence measurements over the range of 60-300 K were performed with a standard closed cycle cryostat set-up. Hydrostatic pressure measurements over the range $0-8.5$ kbar were performed using a gas compressor system. Carrier recombination mechanisms are influenced by the band structure. Conventional methods to change the band structure to investigate

*s.sweeney@surrey.ac.uk; phone +44(0)1483689406; fax +44(0)1483689404;

Novel In-Plane Semiconductor Lasers IX, edited by Alexey A. Belyanin, Peter M. Smowton,

Proc. of SPIE Vol. 7616, 761608 - (c) 2010 SPIE · CCC code: 0277-786X/10/\$18 · doi: 10.1117/12.842253 
the recombination mechanisms, involve growing a series of devices with a range of active region alloy compositions. The high costs involved with growing a lot of wafers, the difficulty in keeping doping densities and layer thicknesses identical mean this method can be unreliable. By using high hydrostatic pressure to reversibly alter the band-structure of one device, the recombination mechanisms can be quickly and easily resolved. The application of high hydrostatic pressure causes an increase in the direct band gap, mimicking the effect of alloying thereby allowing one to investigate wavelength dependent properties of semiconductor lasers. Furthermore, due to the different pressure coefficients of the layers it can also be used to alter the alignment between direct $(\Gamma)$ and indirect $(\mathrm{X}, \mathrm{L})$ ands and to alter the band offsets between layers of differing composition [4]. In order to quantify the different carrier recombination processes, we also measured the pure spontaneous emission from the lasers. Since the light emitted from the facets is strongly influenced by gain/loss as the optical field propagates along the cavity, we instead measure the light emitted in a perpendicular direction. To do this we milled a window in the n-side of the devices using a focused ion beam and aligned an optical fibre with the window to collect the spontaneous emission where the coupling efficiency is kept constant. This experiment was performed as a function of temperature. Further details of this technique are described elsewhere [8].

\section{TEMPERATURE DEPENDENCE}

The threshold current of a semiconductor laser is the point at which the gain equals all of the losses in the device and above this current efficient stimulated emission dominates. It is therefore desirable to have a low (ideally zero) and temperature independent $I_{\text {th }}$ [4]. Figure- 1 shows the integrated spontaneous emission $\left(L_{\text {spon }}\right)$ versus current at $60 \mathrm{~K}$ and $280 \mathrm{~K}$. The two curves have been normalized to the value of $L_{\text {spon }}$ at threshold $\left(=L_{p i n}\right)$, so that the sub threshold shape of both curves can be easily compared and contrasted. The linear behavior of the $L_{\text {spon }}$ versus current curve at $60 \mathrm{~K}$ suggests that the primary current path flowing through the laser may be associative radiative recombination. The non- linearity of the curve down to the lowest currents suggests that some defect related recombination may be present in this material. On the other hand, the sub-linear behavior of the $L_{s p o n}$ versus current curve at $280 \mathrm{~K}$ suggests that a non-radiative process is present and that the process has a stronger dependence on the carrier density than radiative current.

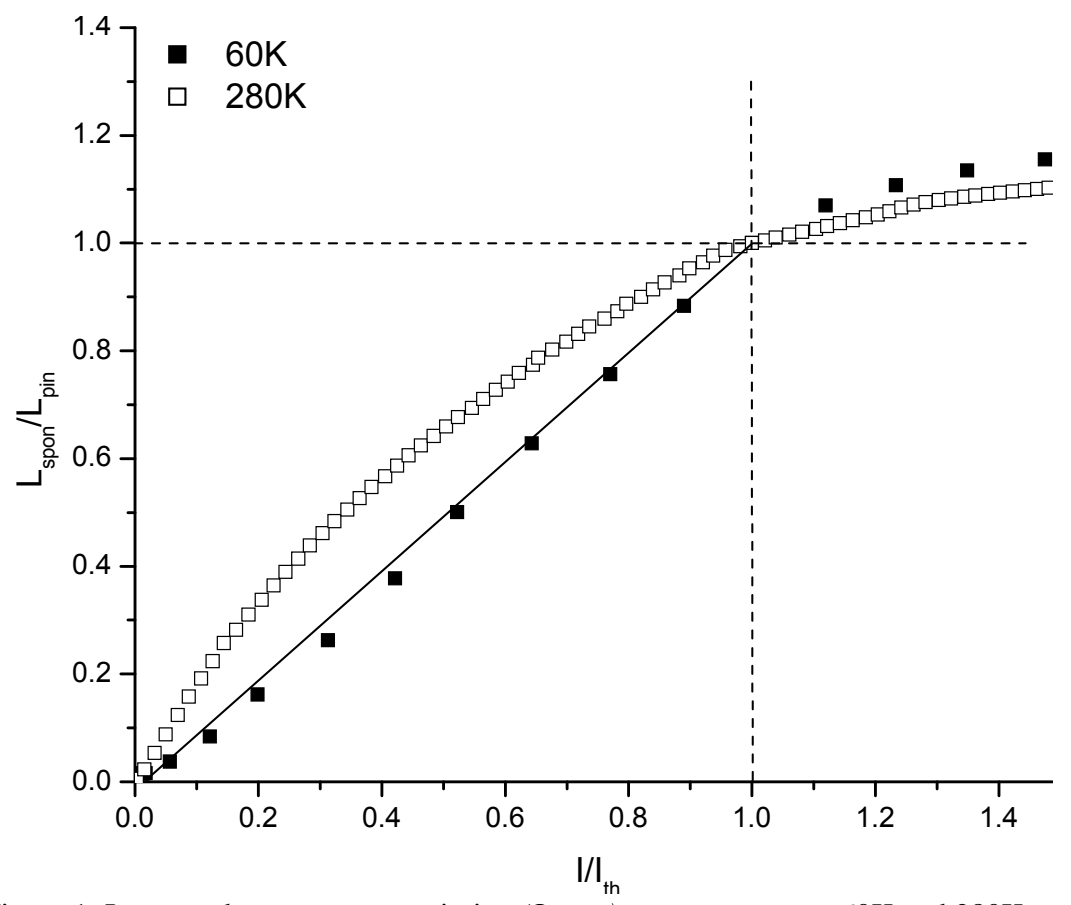

Figure 1: Integrated spontaneous emission $\left(L_{\text {spon }}\right)$ versus current at $60 \mathrm{~K}$ and $280 \mathrm{~K}$.

Figure - 2 shows the normalized (at $T=12 \mathrm{~K}$ ) temperature dependence of the threshold current density, $J_{t h}$ (full squares). We find that the device has a characteristic temperature $\left(T_{0}\right)$ of $88 \mathrm{~K}$ between 12 and $220 \mathrm{~K}$ and $T_{0}=51 \mathrm{~K}$ between 240 and 
$300 \mathrm{~K}$ (near room temperature). From these results we find that the device has similar $T_{0}$ values to conventional InGaAsP/InP devices (typically $\sim 50-60 \mathrm{~K}$ near room temperature). Figure - 2 also shows the temperature dependence of the radiative current density, $J_{\mathrm{rad}}$, (open squares in the figure) found from the pinning level of the integrated spontaneous emission has almost ideal QW linear temperature dependence (inset) leading to the conclusion that the loss mechanisms must increase superlinearly with increasing temperature. From these results we estimate that at 300K the radiative recombination process accounts for up to $\sim 16 \%$ of the total threshold current. Thus, a non-radiative process dominates contributing to $\sim 84 \%$ of $J_{t h}$ at room temperature. From this result it can be also seen that this device has a higher proportion of radiative recombination compared to that reported in [1]. We measured room temperature $J_{\text {th }}$ to be $\sim 253$ $\mathrm{Acm}^{-2}$, which is significantly less than previously obtained values of $\sim 1.6 \mathrm{kAcm}^{-2}$ for GaAsSb/GaAs based $\sim 1.3 \mu \mathrm{m}$ laser[1, 4].

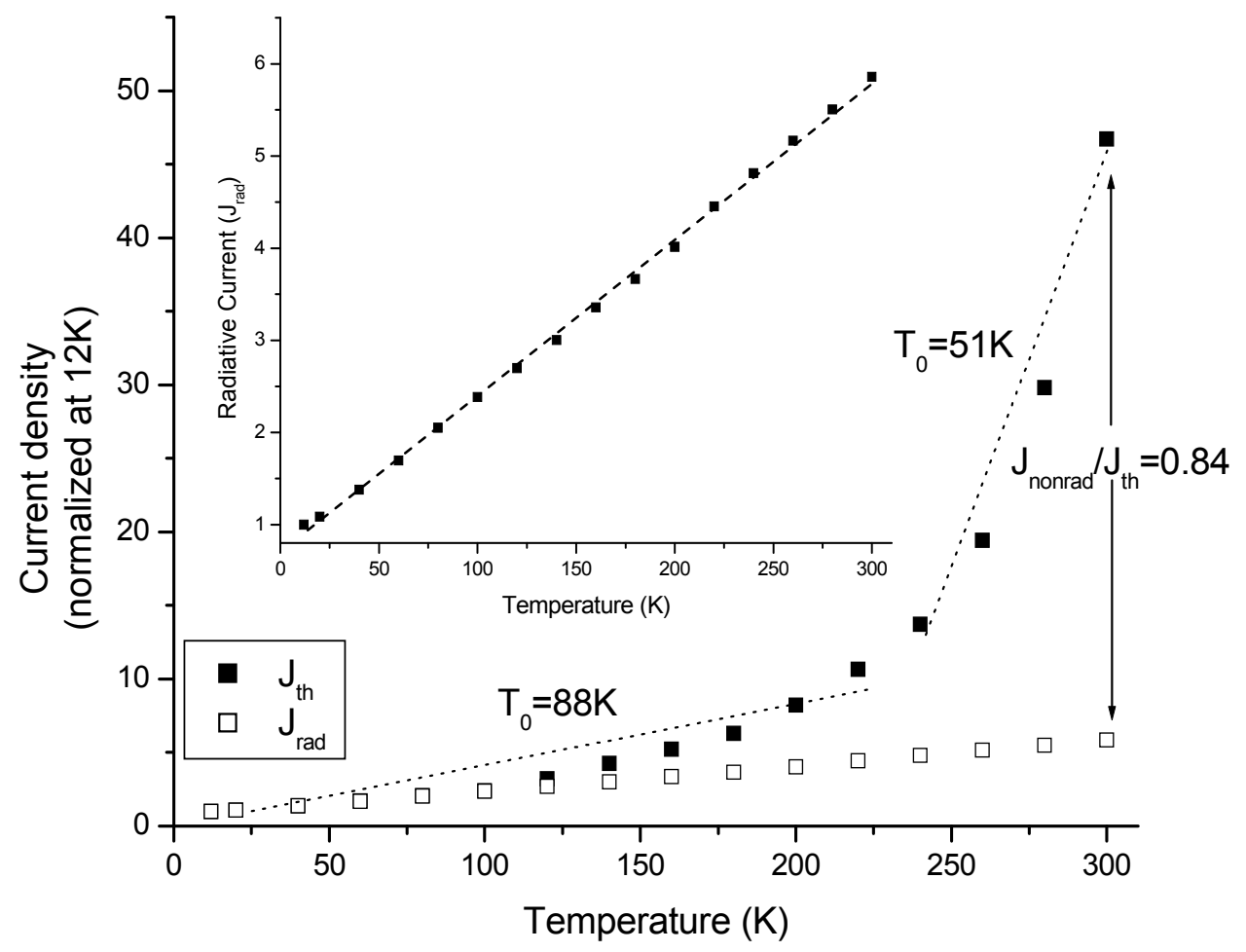

Figure 2: Normalized (at $T=60 \mathrm{~K}$ ) temperature dependence of the threshold current density, $J_{t h}$ (full squares). Temperature dependence of the radiative current density, $\mathrm{J}_{\mathrm{rad}}$, (open squares in the figure) has almost ideal QW linear temperature dependence (inset).

Figure-3 shows the inverse of differential quantum efficiency against the cavity length at RT. The internal quantum efficiency and internal optical loss are calculated as $71 \%$ and $18 \mathrm{~cm}^{-1}$ respectively.

\section{PRESSURE DEPENDENCE}

Figure - 4 shows the measured room temperature pressure dependence of $J_{t h}$. Also shown is the ideal expected variation of $J_{r a d} \alpha E_{g}^{2}$ [9], where $E_{g}$ is the bandgap (taken from $E_{g}=h c / \lambda$, where $\lambda$ is the measured lasing wavelength). $\mathrm{J}_{\text {th }}$ and $J_{\text {rad }}$ have been normalized to their respective values at atmospheric pressure. The $\lambda$ is measured for a strain compensated GaAsSb/GaAs SQW device. Our calculated pressure co-efficient based on measured $\lambda$ is 7.7 meV/kbar, which is approximately the same as previously measured $7.6 \mathrm{meV} / \mathrm{kbar}$ for strain compensated GaAsSb/GaAs 3QW device [1, 4]. Preliminary pressure measurements suggest that the pressure dependence of $J_{\text {rad }}$ in this material system is very close to ideal [10]. From figure - 4, it can be seen that $J_{\text {th }}$ decreases slightly with respect to pressure up to 


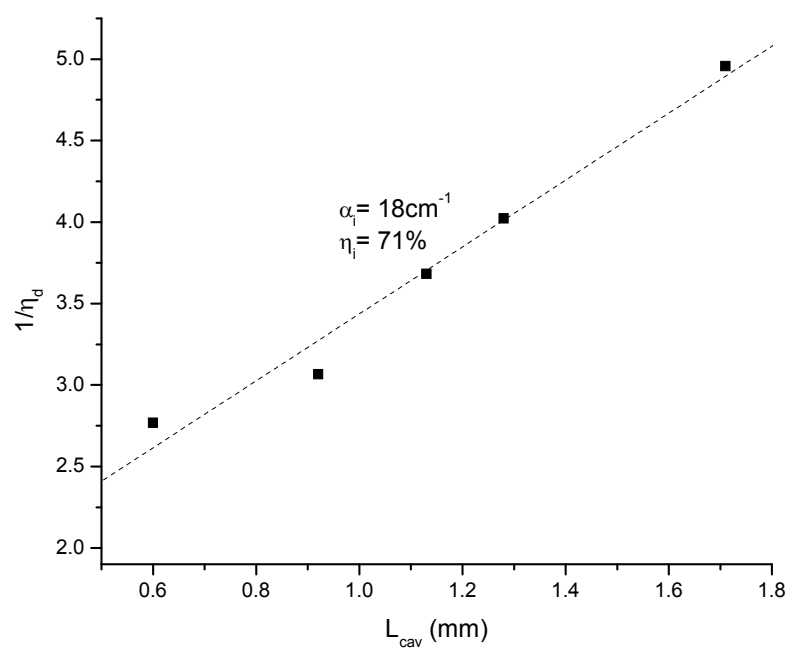

Figure 3: Inverse of differential quantum efficiency $\left(\eta_{d}\right)$ against cavity length $(\mathrm{mm})$ at the room temperature.

$1.5 \mathrm{kbar}$ and then starts to increase. The pressure dependence of the threshold current is stronger, particularly above 6 kbar. This is perhaps unsurprising since, as shown earlier, $\mathrm{I}_{\mathrm{rad}}$ accounts for only $\sim 16 \%$ of $I_{\text {th }}$ at room temperature and atmospheric pressure. From this we conclude that both the slight initial decrease and strong increase in $J_{t h}$ above 6 kbar with pressure is due to a non-radiative process. From temperature dependence measurements we concluded that some defect related recombination is present in this material. The strong change in $J_{t h}$ with pressure suggests that defect related recombination may not dominant. For this reason, the possible non-radiative processes are Auger recombination and carrier leakage. Auger recombination decreases with increase in pressure. This perhaps explains the presence of Auger in this device as $J_{t h}$ decreases by $\sim 5 \%$ with pressure up to $1.5 \mathrm{kbar}$.

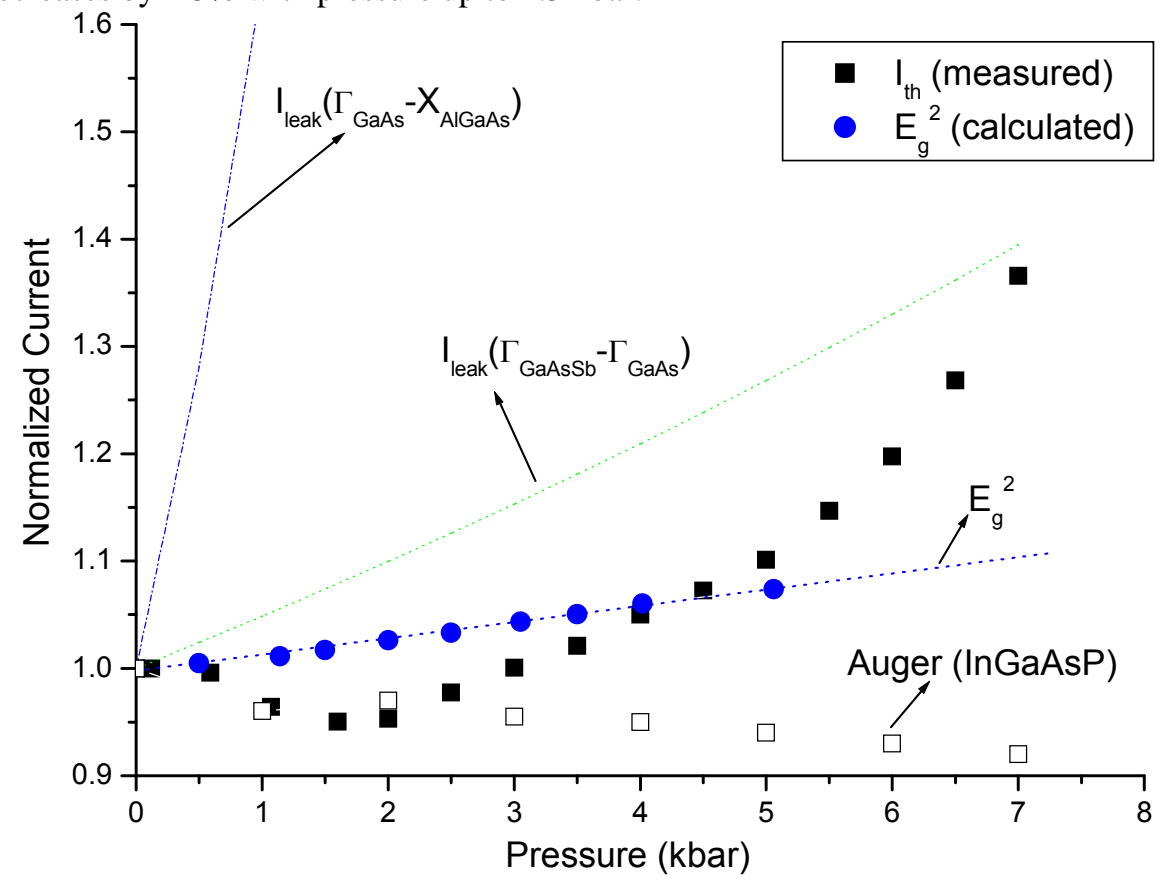

Figure 4: Room temperature pressure dependence of threshold current density (circles). Also shown is the ideal expected variation of radiative current (rectangle), and pressure variations of carrier leakage into $\Gamma_{\mathrm{GaAs}}$ (dot-dot) and $\mathrm{X}_{\mathrm{AlGaAs}}$ (dash-dash). For comparison, the pressure dependence of $\mathrm{I}_{\mathrm{th}}$ for a $1.3 \mu \mathrm{m}$ InGaAsP device (extracted from [1]).

The conduction band offset of the GaAsSb/GaAs interface (for 30\% Sb) is still a matter of debate with reports in the literature of both weak type I [11] and type II alignments [12]. This indicates that the conduction band offset is close to 
zero. From a simple linear interpolation of the band gap pressure coefficients of the constituent binaries, GaAs $\Gamma$ minimum (+10.7 meV/kbar) and GaSb $\Gamma$-minimum (+14.2 meV/kbar) we would expect a shift for the GaAsSb QWs $\Gamma$ minimum of $\sim+11.9 \mathrm{meV} / \mathrm{kbar}$. Due to the slightly larger pressure coefficient of the $\mathrm{GaAs}_{0.7} \mathrm{Sb}_{0.3} \mathrm{QW} \Gamma$-minimum compared to the GaAs barrier $\Gamma$-minimum (difference $=+1.2 \mathrm{meV} / \mathrm{kbar}$ ), occupation of electrons in the GaAs barrier states may increase with increasing pressure, as shown by the calculated dot-dot line in Figure -4 . Furthermore, due to the small or possibly negative (type II) GaAsSb/GaAs band offset, the electrons may also be confined in the GaAs spacer (recombination occurs by cross-interface transitions) and AlGaAs layers is the barrier layer. The opposite pressure coefficient of GaAs spacer $\Gamma$-minimum ( $+10.7 \mathrm{meV} / \mathrm{kbar})$ and AlGaAs barrier X-minimum $(-0.8 \mathrm{meV} / \mathrm{kbar})$ causes the Xminima in the AlGaAs layer and $\Gamma$-minimum of GaAs layer move closer to each other with increasing pressure and may provide an additional leakage path, as shown by the dash-dash line in Figure - 4. Whilst carrier leakage into the GaAs and/or AlGaAs barrier layers offers a possible explanation for the increase of $J_{t h}$ with pressure, it is clear that this in itself is insufficient. The lower rate of increase of $J_{t h}$ with pressure dependence compared to the leakage paths may be explained in carrier leakage together with a non-radiative process that decreases with pressure are both present. This also justifies the findings in Figure- 4 of the presence of Auger recombination in this material.

\section{CONCLUSIONS}

In summary, from our studies we find that these GaAsSb devices have an improved $J_{t h}$ although at room temperature the threshold current remains dominated by nonradiative recombination. The nonradiative process is also responsible for the poor temperature sensitivity of the devices resulting in low $T_{0}$ values around room temperature. From our pressure dependence measurements, we observe that the threshold current shows a slight decrease followed by strong increases with pressure. This suggests that carrier overflow into the GaAs and/or AlGaAs barrier layers together with Auger recombination forms a large nonradiative current path in these devices at normal operating temperatures.

Acknowledgement: The authors gratefully acknowledge Kawn trust, Advanced Technology Institute and Royal Academy of Engineering for financial support.

\section{REFERENCES}

1. K. Hild, S. J. Sweeney, S. Wright, D. A. Lock, S. R. Jin, I. P. Marko, S. R. Johnson, S. A. Cha-parro, S.-Q. Yu, and Y.-H. Zhang, Applied Physics Letters, 89, 173509-173511 (2006).

2. Y. K. Su, C. T. Wan, R. W. Chuang, C. Y. Huang, W. C. Chen, Y. S. Wang and H. C. Yu, Journal of Crystal Growth, 310, 4850-4853 (2008).

3. S.-Q. Yu, X. Jin, S. R. Johnson and Y.-H. Zhang, Journal of Vacuum Science and Technology B, 24(3), 1617-1621 (2006).

4. K. Hild, S. J. Sweeney, I. P. Marko, S. R. Jin, S. R. Johnson, S. A. Chaparro, S.-Q Yu, and Y-H. Zhang, Physics Status Solidi B, 244(1), 197-202 (2007).

5. G. Blume, T.J.C Hosea, S.J. Sweeney, S.R. Johnson, J.-B. Wang and Y.-H. Zhang, IEE Proc.-Optoelectron., 152(2), 110-117 (2005).

6. P. Dowd, S. R. Johnson, S. A. Fe1d, M. Adamcyk, S. A. Chaparro, J. Joseph, K. Hilgers, M.P. Homing, K. Shiralagi and Y.-H. Zhang, Electronics Letter, 39(13), 987-988 (2003).

7. J.-B. Wang, S. R. Johnson, S. A. Chaparro, D. Ding, Y. Cao, Yu. G. Sadofyev, Y.-H. Zhang, J. A. Gupta and C. Z. Guo, Physical Review B, 70, 195339 1- 1953398 (2004).

8. S. J. Sweeney, A. F. Philips, A. R. Adams, E. P. O’Reilly, and P. J. A. Thijs, IEEE Photonics Technol. Lett. 10, 1076 (1998).

9. A.R. Adams, M. Silver and J. Allam, Semiconductors and Semimetals, 55, 301 (1998).

10. K. Hild, S. J. Sweeney, D. A. Lock, S. Wright, J.-B Wang, S. R. Johnson and Y-H. Zhang, Lasers and ElectroOptics Annual Meeting (LEOS 2005), Pages 331-32, Sydney, Australia, October 2005.

11. S. R. Johnson, C. Z. Guo, S. Chaparro, Yu. G. Sadofyev, J. Wang, Y. Cao, N. Samal, J. Xu, S. Q. Yu, D. Ding, and Y.-H. Zhang, J. Cryst. Growth 251, 521 (2003).

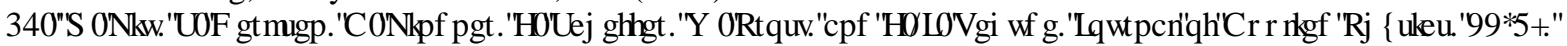

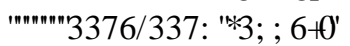

